\title{
On the Unavoidable Uncertainty of Truth in Dynamic Geometry Proving
}

\author{
Francisco Botana and Tomas Recio
}

\begin{abstract}
The aim of this note is to discuss some issues posed by the emergency of universal interfaces able to decide on the truth of geometric statements. More specifically, we consider a recent GeoGebra module allowing general users to verify standard geometric theorems. Working with this module in the context of Varignon's theorem, we were driven - by the characteristics of the GeoGebra interface - to perform a quite detailed study of the very diverse fate of attempting to automatically prove this statement, when using two different construction procedures. We highlight the relevance -for the theorem proving output- of expression power of the dynamic geometry interface, and we show that the algorithm deciding about the truth of some -even quite simplestatements can fall into a not true and not false situation, providing a source of confusion for a standard user and an interesting benchmark for geometers interested in discovering new geometric facts.
\end{abstract}

Mathematics Subject Classification (2010). Primary 68T15; Secondary 68W30.

Keywords. Dynamic geometry, automated theorem proving, GeoGebra, Varignon Theorem.

\section{Introduction}

Since Sept. 2014, it is the first time a well spread, dynamic geometry program, GeoGebra, with over 20 million users (many of them related to the educational community) all around the world, includes a (yet quite novice) automatic theorem proving feature. It is, therefore, also the first time that some achievements from a half century history of automated deduction in geometry (ADG) research, are actually exposed to a global customer through an open use, moving away from university labs and controlled learning experiments.

The GeoGebra Theorem Proving feature can be roughly described as follows. Each of the steps of a geometric construction (built in the dynamic geometry style, by using different tools from the program) are internally translated into algebraic terms, by following an a priori programestablished geometry/algebra dictionary. Next, on this construction, the user can formulate different queries (are collinear points $X, Y, Z$ ?, are parallel lines $r, s ?, \ldots$ and the like) from a given list of relation questions, that are also automatically translated by the program into algebraic terms. Then, following different criteria, constraints and heuristics, a collection of algebraic methods in ADG, some of them using the own GeoGebra symbolic computation features, some others connecting with an external server (see, for instance, [1]) are activated and sequentially attempt dealing with the proposed question, until one of them eventually succeeds or the program yields a failure warning. In the successful case, the output is a grant/denial of the truth of the proposed statement, eventually including a list of non-degeneracy conditions for the validity of the proposition. Further details are provided in [2]. 
Moreover, it can be remarked that most of the algebraic methods implemented in the GeoGebra Prover portfolio follow an approach and terminology that has become standard after Chou [3]. In Section 2 we present a summary of the main definitions and results in this framework, as described in Recio and Vélez [5].

An interesting issue in this context, and one of the motivations for this paper, has to do with the existence of some statements that are neither generally true nor generally false (following the terminology of [5]). Roughly speaking, statements dealing with constructions that have multiple instances for a single value of the free parameters of the corresponding construction and which are neither true for all such instances nor false for all of them. Section 3 introduces a simple illustrative example ${ }^{1}$, where a statement, neither generally true nor generally false (if naively formulated), can be easily turned into a generally true one, by adding an "intuitive" and natural complementary hypothesis. Furthermore, it advances some proving related consequences when defining, in dynamic geometry, the midpoint of a segment.

Finally, Section 4 deals in detail with one particularly hard example: Varignon's theorem (Subsection 4.1) and its converse (Subsection 4.2). Both statements deal with properties of midpoints of some segments and it turns that, depending on some precise formulation of the concept of midpoint, the statements can greatly vary in many different aspects: truth, computing time, etc. (see Table 2). In fact, one of the formulations (Subsection 4.2.2) leads to a Converse Varignon theorem that is neither true nor false. This example also shows that -without performing a primary decomposition, something out of the scope of most dynamic geometry programs with automatic proving features- it is quite non trivial to guess complementary conditions to break off such undesirable status of confusion (not true, not false). It is, also, an opportunity for humans (such as graduate students!) to use effective algebraic geometry tools to attempt discovering such complementary conditions (as achieved in Subsection 4.2.3.).

As remarked above, all these reflections -on the hidden subtleties of the topic- can be specially interesting nowadays, in view of the current trend concerning the inclusion of proving features in dynamic geometry programs.

\section{Short survey of some automatic theorem proving key concepts}

Let $H\left(x_{1}, \ldots, x_{n}\right)$ denote the collection of equations describing the hypotheses of a statement and let $T\left(x_{1}, \ldots, x_{n}\right)$ be the (single) thesis (the required modifications for the case of several simultaneous theses are quite straightforward). Let $I=(H, T * z-1)$ be the ideal of hypotheses and negated thesis in $K\left[x_{1}, \ldots, x_{n}, z\right]$, where $K$ is an algebraically closed field.

Definition 2.1. Let $\left\{x_{1}, \ldots, x_{n}\right\}$ be the collection of coordinates involved in the algebraic description of the hypotheses, with $\left\{x_{1}, \ldots, x_{r}\right\}$ taken as a maximum-size set of free variables for the hypotheses.

This means:

a) the dimension (HilbertDimension) of the ideal of hypotheses is $r$, and

b) the elimination in the hypotheses ideal of all the variables minus $\left\{x_{1}, \ldots, x_{r}\right\}$ yields 0 (i.e., these variables are free modulo $H$ ).

Both conditions imply that:

i) there is no polynomial relation in the ideal of hypotheses holding for the variables $\left\{x_{1}, \ldots, x_{r}\right\}$ alone, and

ii) $r$ is the largest number of variables having that property with respect to the ideal of hypotheses (because of the concept of Ideal Dimension). Therefore, for any extra variable $x_{m}$, with $m>r$,

\footnotetext{
${ }^{1}$ Kept specially simple in this Section because the purpose here is merely introductory, but one can show it hides some unexpected complications.
} 
the elimination with respect to $\left\{x_{1}, \ldots, x_{r}, x_{m}\right\}$ is not 0 , i.e. there is at least one non zero polynomial in the variables $\left\{x_{1}, \ldots, x_{r}, x_{m}\right\}$ belonging to the ideal $H$ (see several detailed comments on this issue in Recio, Sterk and Vélez [6]).

Now, let us project the variety $V=V(H, T * z-1) \subseteq K^{n+1}$ over the affine space of free variables $K^{r}$. Then, the Zariski closure of the projection $\pi(V)$ is the zero set $V_{r} \subseteq K^{r}$ of the elimination ideal $I_{r}=(H, T * z-1) \cap K\left[x_{1}, \ldots, x_{r}\right]$. Let $J$ be the ideal $(H, T)$ in $K\left[x_{1}, \ldots, x_{n}\right]$ and let $W$ be its zero set. Let $J_{r}$ be the elimination ideal $J_{r}=(H, T) \cap K\left[x_{1}, \ldots, x_{r}\right]$ and let $W_{r}$ be its zero set in $K^{r}$.

Definition 2.2. The statement $H \Longrightarrow T$ is said to be generally true if $I_{r} \neq 0$, and generally false if $J_{r} \neq 0$.

It should be remarked that in the generally true case, the lifting of points in $V_{r}$ to the zero set of $H$ provides instances of the hypotheses where the statement fails; that is, values of $\left(x_{1}, \ldots, x_{r}\right)$ in $I_{r}$ such that there is a value $\left(x_{r+1}, \ldots, x_{n}\right)$ verifying $H$ and not $T$ (but it could be that also, for the same value of $\left(x_{1}, \ldots, x_{r}\right)$, there is a different value $\left(x_{r+1}, \ldots, x_{n}\right)$ verifying $H$ and $\left.T\right)$. Obviously, the irreducible components of $H$ yielding values where it holds that there are values of $\left(x_{1}, \ldots, x_{r}\right)$ in $I_{r}$ such that there is a value $\left(x_{r+1}, \ldots, x_{n}\right)$ verifying $H$ and not $T$, are irreducible components of $H$ where the variables $\left(x_{1}, \ldots, x_{r}\right)$ do not remain independent (since the elements of $I_{r}$ belong to this component). Thus, they are labeled as degenerate, since it is implicit some kind of intuition that geometrically sound constructions should be those where $\left(x_{1}, \ldots, x_{r}\right)$ are free... Same kind of reflection can be considered for the generally false case.

Finally we should recall that a statement is generally true iff the thesis holds over all irreducible components of the hypotheses variety that are non-degenerate: i.e. such that $\left(x_{1}, \ldots, x_{r}\right)$ remain independent modulo this irreducible component. It is generally false iff the thesis does not hold over any of the non-degenerate components [4, Propositions 1 and 2]. Both generally true and generally false cannot simultaneously happen, as it can be derived from the following proposition.

Proposition 2.3. If a statement is generally true (resp. false), then it is not generally false (resp. true). In symbols, $I_{r} \neq 0 \Longrightarrow J_{r}=0$, and $J_{r} \neq 0 \Longrightarrow I_{r}=0$.

Proof. It is enough to show that it can not simultaneously happen generally true and generally false.

Let us prove that $J_{r}$ and $I_{r}$ can not be simultaneously not zero. In fact, assume they are both zero and let $g \in(H, T), q \in(H, T * z-1)$ be both non zero elements of $K\left[x_{1}, \ldots, x_{r}\right]$.Thus,

$$
\begin{gathered}
g=\text { combination of } H+\text { multiple of } T, \\
q=\text { combination of } H+\text { multiple of }(T * z-1),
\end{gathered}
$$

where combination of $H$ is a way of expressing a sum of polynomials in $n$ variables times elements of $H$; multiple of $T$ (resp. $(T * z-1)$ ) is a way of expressing a polynomial in $n$ variables times $T$ (resp. a polynomial in $n+1$ variables times $(T * z-1)$ ).

Replacing $z$ by $1 / T$ and multiplying by a suitable power of $T$, say $T^{m}$, the last equality turns to be

$$
q * T^{m}=\text { combination of } H .
$$

Analogously, the expression of $g$ above can be rewritten as

$$
\text { multiple of } T=\text { combination of } H-g \text {. }
$$

Thus,

$$
(\text { multiple of } T)^{m}=(\text { combination of } H-g)^{m}=\text { combination of } H+g^{m},
$$


and hence

$$
q *(\text { multiple of } T)^{m}=q * \text { combination of } H+q * g^{m} .
$$

Since $q *(\text { multiple of } T)^{m}=$ multiple of $q * T^{m}=$ combination of $H$, finally we arrive to combination of $H-q *$ combination of $H=$ combination of $H=q * g^{m}=$

$$
=\text { non zero element of } K\left[x_{1}, . ., x_{r}\right],
$$

which is impossible, since we assume $x_{1}, . ., x_{r}$ to be free variables for $\mathrm{H}$.

Thus, we see that both elimination ideals can not be simultaneously different from zero. Table 1 summarizes the possibilities.

\begin{tabular}{|l|c|c|}
\hline & $J_{r}$ & $I_{r}$ \\
\hline not generally true and not generally false & $(0)$ & $(0)$ \\
\hline generally true (and, thus, not generally false) & $(0)$ & Not $(0)$ \\
\hline generally false (and, thus, not generally true) & Not $(0)$ & $(0)$ \\
\hline
\end{tabular}

Table 1. Summary of possibilities of being generally true or false

Moreover, neither not generally true does imply being generally false, nor not generally false does imply being generally true, since there are examples of statements that are simultaneously not generally true and not generally false. A nice example is, precisely, the converse of Varignon (in the case of a particular algebraic interpretation of the concept of midpoint, see Subsection 4.2).

Thus, in order to decide if a statement is generally true or not generally true (beware, this is not the same as being generally false), all we have to do is to establish a procedure for deciding, given a polynomial ideal (of hypotheses and negated theses), whether the result of eliminating in the ideal some variables, yields the zero ideal or not.

In conclusion, we identify the concept of proving a statement with proving that it is generally true. If it is not, then we would like to learn if it is generally false (roughly speaking, false everywhere it makes sense) or if it is, on the contrary, true over some relevant components and false over some other relevant components, thus showing that there is some hidden important fact holding in some special cases to be discovered with further computations and insight! See the second formulation of the converse of Varignon in Subsection 4.2.

\section{A simple example}

Let us consider the following construction: Given two points $A\left(t_{1}, t_{2}\right), B\left(t_{3}, t_{4}\right)$, construct circle $c_{1}$ with center $A$ and going through $B$, and circle $c_{2}$ with center $B$ and going through $A$. Then, consider the intersection of $c_{1}$ and $c_{2}$, points $E\left(e_{1}, e_{2}\right)$ and $F\left(f_{1}, f_{2}\right)$.

Clearly the construction depends only on $t_{1}, t_{2}, t_{3}, t_{4}$, but $E$ and $F$ are not uniquely determined, since they are described as the solution of the system

$$
\left.\left(x-t_{1}\right)^{2}+\left(y-t_{2}\right)^{2}-\left(t_{3}-t_{1}\right)^{2}-\left(t_{4}-t_{2}\right)^{2}\right),\left(x-t_{3}\right)^{2}+\left(y-t_{4}\right)^{2}-\left(t_{1}-t_{3}\right)^{2}-\left(t_{2}-t_{4}\right)^{2} .
$$

The expression of the coordinates for $E, F$, through the Maple RootOf function expressing the solutions of a second degree univariate polynomial equation, shows that there are two different, indistinguishable solutions: 


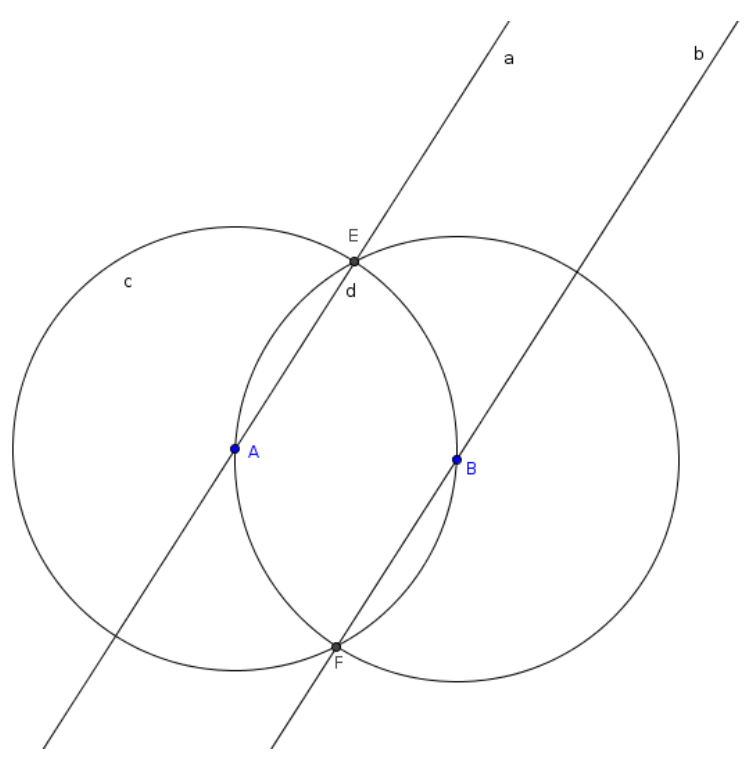

FIGURE 1. A simple example

$>$ solve $\left(\left\{(x-t 1)^{\wedge} 2+(y-t 2)^{\wedge} 2-\left((t 3-t 1)^{\wedge} 2+(t 4-t 2)^{\wedge} 2\right),(x-t 3)^{\wedge} 2+(y-t 4)^{\wedge} 2\right.\right.$ $\left.\left.-\left((t 1-t 3)^{\wedge} 2+(t 2-t 4)^{\wedge} 2\right)\right\},\{x, y\}\right)$;

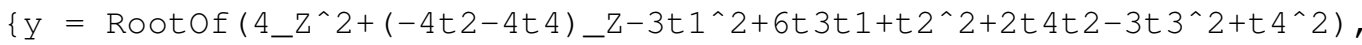
$x=-(1 / 2)\left(2 \operatorname{Rootof}\left(4-z^{\wedge} 2+(-4 t 2-4 t 4) z^{2}-3 t 1^{\wedge} 2+6 t 3 t 1+t 2^{\wedge} 2+2 t 4 t 2\right.\right.$

$\left.-3 t 3^{\wedge} 2+t 4^{\wedge} 2\right) t 2-2 \operatorname{Rootof}\left(4-z^{\wedge} 2+(-4 t 2-4 t 4)-z-3 t 1^{\wedge} 2+6 t 3 t 1+t 2^{\wedge} 2+\right.$ $\left.\left.\left.2 t 4 t 2-3 t 3^{\wedge} 2+t 4^{\wedge} 2\right) t 4-t 1^{\wedge} 2-t 2^{\wedge} 2+t 3^{\wedge} 2+t 4^{\wedge} 2\right) /(t 1-t 3)\right\}$

Anyway, let us suppose a naive (more properly described as standard) user that wants to prove the following thesis:

Line $A E$ ( $a$ in Figure 1) is parallel to line $B F$ (resp. $b$ ).

Surely, this naive user is not going to reflect on this particular lack of determination for $E$ and $F$ in the construction and, thus, he/she might be perplexed to find that the following Maple computation

$>$ with (PolynomialIdeals): EliminationIdeal $\left(<(e 1-t 1)^{\wedge} 2+(e 2-t 2)^{\wedge} 2-(\right.$ $\left.(t 3-t 1)^{\wedge} 2+(t 4-t 2)^{\wedge} 2\right),(e 1-t 3)^{\wedge} 2+(e 2-t 4)^{\wedge} 2-\left((t 1-t 3)^{\wedge} 2+(t 2-t 4)^{\wedge} 2\right)$, $(f 1-t 1)^{\wedge} 2+(f 2-t 2)^{\wedge} 2-\left((t 3-t 1)^{\wedge} 2+(t 4-t 2)^{\wedge} 2\right),(f 1-t 3)^{\wedge} 2+(f 2-t 4)^{\wedge} 2-($ $\left.(t 1-t 3)^{\wedge} 2+(t 2-t 4)^{\wedge} 2\right),((e 1-t 1) *(f 2-t 4)-(e 2-t 2) *(f 1-t 3)) * t-1>$, $\{t 1, t 2, t 3, t 4\}) ;$

$$
<0>
$$

shows that this statement is not generally true, and

$>$ EliminationIdeal $\left(<(e 1-t 1)^{\wedge} 2+(e 2-t 2)^{\wedge} 2-\left((t 3-t 1)^{\wedge} 2+(t 4-t 2)^{\wedge} 2\right),(e 1-\right.$ t 3$)^{\wedge} 2+(e 2-t 4)^{\wedge} 2-\left((t 1-t 3)^{\wedge} 2+(t 2-t 4)^{\wedge} 2\right),(f 1-t 1)^{\wedge} 2+(f 2-t 2)^{\wedge} 2-((t 3-$ t 1$\left.)^{\wedge} 2+(t 4-t 2)^{\wedge} 2\right),(f 1-t 3) \wedge 2+(f 2-t 4)^{\wedge} 2-\left((t 1-t 3)^{\wedge} 2+(t 2-t 4) \wedge 2\right), \quad((e 1$ $-t 1) *(f 2-t 4)-(e 2-t 2) *(f 1-t 3))>,\{t 1, t 2, t 3, t 4\}) ;$ 
shows that it is not generally false, since it is true or false depending on some particular choices for $E, F$.

In fact, by just adding the condition

$$
\left(\left(e_{1}-f_{1}\right) h-1\right)\left(\left(e_{2}-f_{2}\right) s-1\right)=0,
$$

that means

$$
\left(\left(e_{1}-f_{1}\right) h-1\right)=0 \text { or }\left(\left(e_{2}-f_{2}\right) s-1\right)=0,
$$

and this is equivalent to

$$
e_{1} \neq f_{1} \text { or } e_{2} \neq f_{2}
$$

i.e., to

$$
E \neq F \text {. }
$$

i.e. it happens that the statement is generally true, as checked by

$>$ EliminationIdeal $\left(<(e 1-t 1)^{\wedge} 2+(e 2-t 2)^{\wedge} 2-\left((t 3-t 1)^{\wedge} 2+(t 4-t 2)^{\wedge} 2\right),(e 1-\right.$ t 3$)^{\wedge} 2+(e 2-t 4)^{\wedge} 2-\left((t 1-t 3)^{\wedge} 2+(t 2-t 4)^{\wedge} 2\right),(f 1-t 1)^{\wedge} 2+(f 2-t 2)^{\wedge} 2-((t 3-$

t 1$\left.)^{\wedge} 2+(t 4-t 2)^{\wedge} 2\right),(f 1-t 3)^{\wedge} 2+(f 2-t 4)^{\wedge} 2-\left((t 1-t 3)^{\wedge} 2+(t 2-t 4)^{\wedge} 2\right),((e 1$

$-f 1) * h-1) *((e 2-f 2) * s-1),((e 1-t 1) *(f 2-t 4)-(e 2-t 2) *(f 1-t 3)) * t-1>$,

$\{t 1, t 2, t 3, t 4\}) ;$

$$
<t 3-t 1, t 4-t 2>
$$

under the non-degeneracy condition

$$
t_{1} \neq t 3 \text { or } t_{2} \neq t_{4}
$$

or, equivalently,

$$
\operatorname{not}\left(t_{1}=t_{3} \text { and } t_{2}=t_{4}\right) \text {, }
$$

that is,

$$
\operatorname{not}(A=B) \text {. }
$$

As shown in Table 1, then the statement is not generally false in this case:

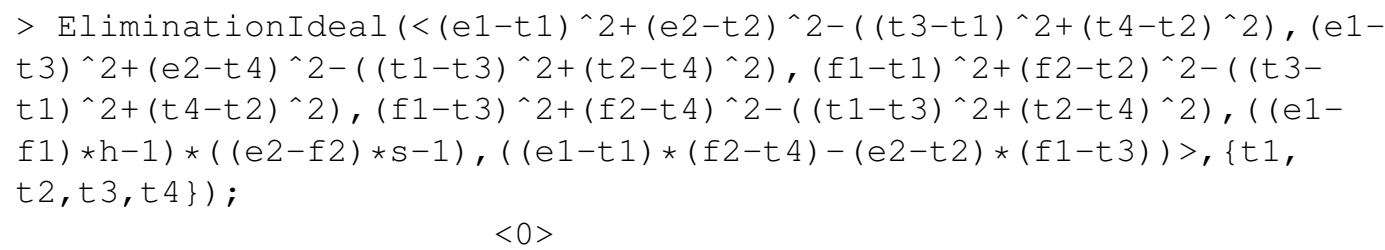

\section{The Varignon theorem and its converse}

The preceding Section highlights -in a deceivingly simple instance- the need to search for complementary and reasonable hypotheses for a given statement to hold generally true or generally false. In that example, it seems quite "intuitive", to require that not $(E=F)$ (and, then, to add as a nondegeneracy condition, that not $(A=B)$ for the statement to be true). But it is not obvious (unless we perform a very costly and usually hard to interpret, geometrically, primary decomposition) at all, in many statements, what could be some of the implicit requirements that should be added to yield a clear conclusion in the given context, i.e., to turn the given the given statement to be generally true or generally false.

This Section introduces one particular illustrative example of this problem: the Varignon theorem (the midpoints of the sides of an arbitrary quadrilateral form a parallelogram) and its converse. Both statements deal with properties of midpoints of some segments. We remark that the direct and converse statements involve, in different ways, the definition of midpoint of a segment. In the direct 
statement we need to describe the midpoint $M$ of some given points $A, B$. In the converse statement, given $M$ and $A$, we need to describe $B$ so that $M$ is the midpoint of segment $A B$.

\begin{tabular}{|c|c|c|}
\hline & Option a) for describing hypotheses & Option b) for describing hypotheses \\
\hline Varignon Direct & $\begin{array}{l}\text { - generally true (in fact, no non- } \\
\text { degeneracy condition) } \\
\text { - easy to compute }\end{array}$ & $\begin{array}{l}\text { - generally true (but with several } \\
\text { non-degeneracy conditions) } \\
\text { - several hours computation of } \\
\text { elimination } \\
\text { - use of alternative, faster but less } \\
\text { canonical algorithm (e.g. Maple's } \\
\text { solve command) requires under- } \\
\text { standing the algebraic geometry } \\
\text { associated to the statement (lift- } \\
\text { ing construction free points to the } \\
\text { hypotheses variety, deciding how } \\
\text { many liftings can be done, deciding } \\
\text { if some/all/none of them verify the } \\
\text { theses) }\end{array}$ \\
\hline Varignon Converse & $\begin{array}{l}\text { - generally true (but with one non- } \\
\text { degeneracy condition) } \\
\text { - easy to compute }\end{array}$ & $\begin{array}{l}\text { - not generally true, not generally } \\
\text { false } \\
\text { - several hours computation of } \\
\text { elimination } \\
\text { - use of alternative, faster but less } \\
\text { canonical algorithm (e.g. Maple's } \\
\text { solve command) requires under- } \\
\text { standing the algebraic geometry } \\
\text { associated to the statement (lift- } \\
\text { ing construction free points to the } \\
\text { hypotheses variety, deciding how } \\
\text { many liftings can be done, deciding } \\
\text { if some/all/none of them verify the } \\
\text { theses) } \\
\text { - this alternate process, requiring } \\
\text { exploring the algebraic geometry } \\
\text { of the involved statement, provides } \\
\text { an excellent benchmark for gradu- } \\
\text { ate students, requiring several chal- } \\
\text { lenging algebra-geometry interpre- } \\
\text { tations and yielding to, perhaps, } \\
\text { new geometric facts }\end{array}$ \\
\hline
\end{tabular}

Table 2. Output of our exploration.

A naive, obvious, treatment of both cases is to consider

a) the coordinates of $M$ as $(A+B) / 2$ in the direct Varignon theorem, and, thus, in the converse case, the coordinates of $B$ as $2 M-A$.

A different option is to consider

b) the midpoint $M$ as a point equidistant of $A$ and $B$ and in the line $A B$; accordingly, point $B$ as the intersection of the line $M A$ with the circle with center $M$ and radius $M A$. 
We might think that it should be evident that the first option is the correct one and the second is by far too artificial. But we have remarked, at the beginning of this note, that we want to consider the case of automated theorem proving (ATP) in the context of the popularization of dynamic geometry software, such as GeoGebra. And, in this particular software, it happens that there is a built-in tool for constructing the midpoint $M$ of a segment $A B$, with algebraic translation $M=(A+B) / 2$, but the determination of $B$ such that $M$ is the midpoint of $A B$ is not yet provided by the program and requires a specific construction, such as building line $M A$ and its intersection with the circle of center $M$ and radius $M A$ through, for instance, GeoGebra's compass tool.

In the following Subsections we explore the consequences, both in the direct and converse Varignon statement, of choosing each of the above mentioned options for describing -in the hypotheses of the direct and the converse Varignon- the midpoint $M$ of some given segment $A B$, and the point $B$ such that a given point $M$ is the midpoint of $A B$, for some other given point $A$.

Let us advance that the output of our exploration is rather diverse, as detailed in Table 2.

Note that the case Converse Varignon | Option b) is the currently natural one for users of ATP tools embedded in dynamic geometry systems such as GeoGebra, but its output (not generally true and not generally false) could be rather disappointing for a standard user. One should not claim that this is a rather artificial example: we have all learned from the past that the algebraic approach to ATP in geometry involves uncontrolled (by the user) problems with the algebraic translations that could rise in the most unexpected contexts. In fact, the origin of this paper has been the search for an answer to what we, not "naive" users at all, obtained when proceeding, in the most direct way, to address the Converse Varignon statement with the current GeoGebra Proving tool.

\subsection{The Direct Varignon theorem}

Using option a) for the concept of midpoint $M$ between $P$ and $Q$ as the point $M=(P+Q) / 2$, that is, the coordinates of $Q$ are those of $M+(M-P)$, consider a quadrilateral $A(0,0), B(1,0), C\left(t_{1}, t_{2}\right)$, $D\left(d_{1}, d_{2}\right)$, and the side midpoints $E\left(t_{3}, t_{4}\right), F\left(f_{1}, f_{2}\right), I\left(i_{1}, i_{2}\right), K\left(k_{1}, k_{2}\right)$. The construction is a 4-dimensional one, with free variables the coordinates of $C, D$, and the hypotheses ideal is

$$
\left.<2 t_{3}-1, t_{4}, 2 f_{1}-t_{1}-1,2 f_{2}-t 2,2 i_{1}-t_{1}-d_{1}\right), 2 i_{2}-t_{2}-d_{2}, 2 k_{1}-d_{1}, 2 k 2-d_{2}>\text {. }
$$

The theses state that EFIK is a parallelogram (Figure 2), that is,

$$
\begin{gathered}
E F \| I K:\left(f_{1}-t_{3}\right)\left(k_{2}-i_{2}\right)-\left(f_{2}-t_{4}\right)\left(k_{1}-i_{1}\right)=0, \text { and } \\
E K \| F I:\left(k_{1}-t_{3}\right)\left(f_{2}-i_{2}\right)-\left(k_{2}-t_{4}\right)\left(f_{1}-i_{1}\right)=0 .
\end{gathered}
$$

The elimination of the free variables $\left(t_{1}, t_{2}, d_{1}, d_{2}\right)$ in the ideal of hypotheses and negation of the theses

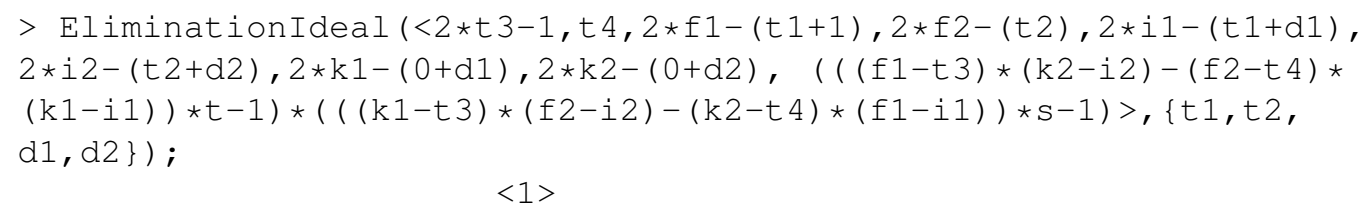

shows that the only conditions for the statement to be false is $1=0$, that is, never. It is generally true, each of the thesis is a combination of the hypotheses:

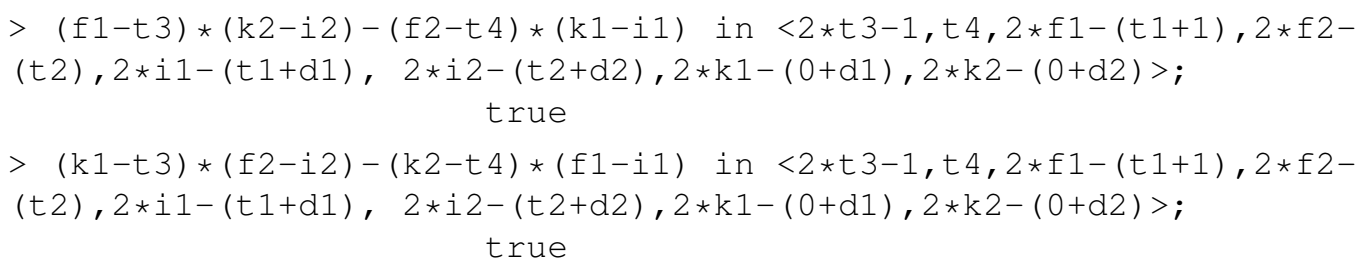




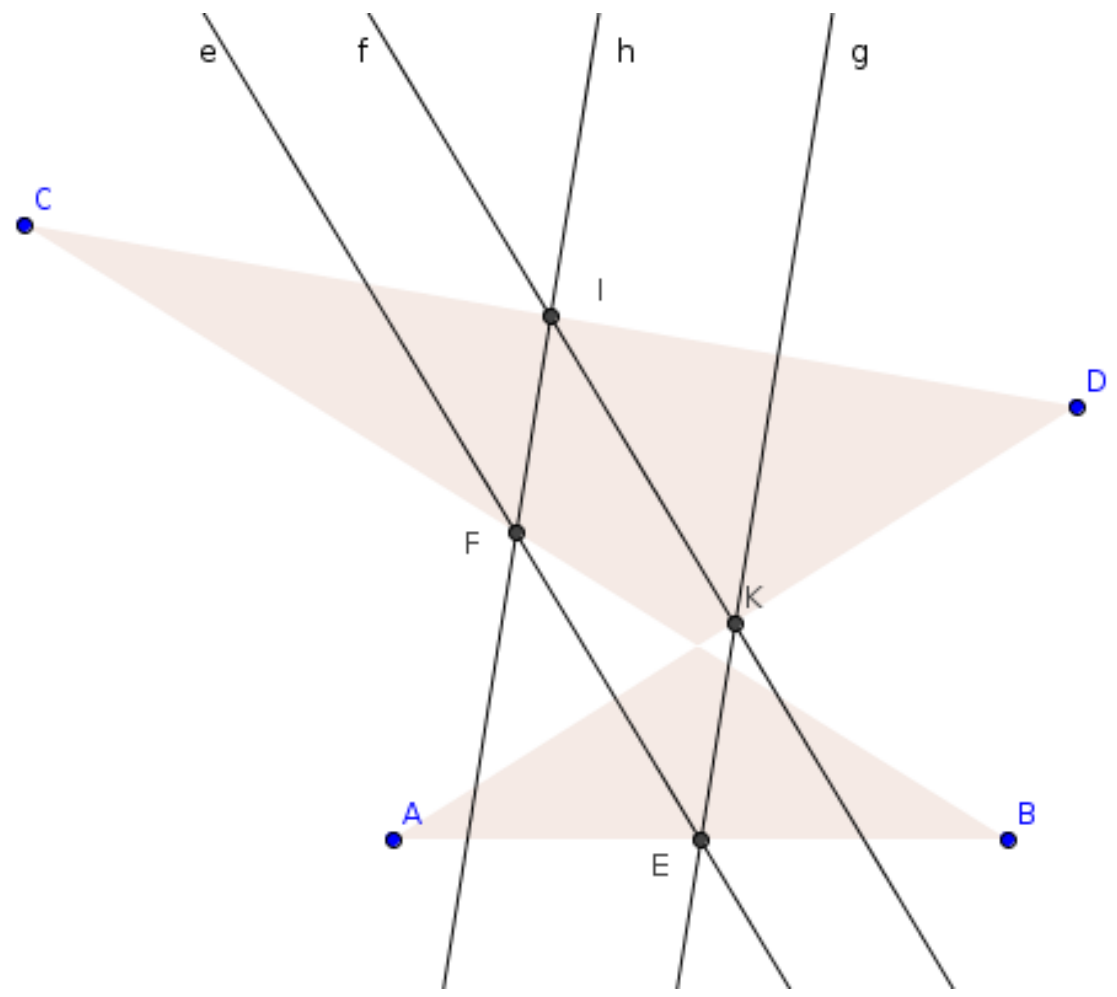

FIGURE 2. Varignon theorem for a non convex quadrilateral

Now, using the concept of midpoint $M$ between $P$ and $Q$ as the center $M$ of a circle of radius $M P$ that intersects the line $M P$ in the other point $Q$, the ideal of hypotheses is

$<t_{3}^{2}-\left(1-t_{3}\right)^{2},\left(1-f_{1}\right)^{2}+f_{2}^{2}-\left(t_{1}-f_{1}\right)^{2}-\left(t_{2}-f_{2}\right)^{2},\left(t_{1}-i_{1}\right)^{2}+\left(t_{2}-i_{2}\right)^{2}-\left(d_{1}-i_{1}\right)^{2}-\left(d_{2}-i_{2}\right)^{2}$, $\left(d_{1}-k_{1}\right)^{2}+\left(d_{2}-k_{2}\right)^{2}-k_{1}^{2}-k_{2}^{2}, t_{4},\left(1-f_{1}\right)\left(t_{2}-f_{2}\right)+f_{2}\left(t_{1}-f_{1}\right),\left(d_{1}-i_{1}\right)\left(t_{2}-i_{2}\right)-\left(d_{2}-i_{2}\right)\left(t_{1}-i_{1}\right)$, $-\left(d_{1}-k_{1}\right) k_{2}+\left(d_{2}-k_{2}\right) k_{1}>$.

It is again a 4-dimensional construction, with free variables the coordinates of $C, D$. Solving respect to the free variables shows that for every position of $C, D$, there is a unique value of the depending variables:

$t_{4}=0, f_{2}=\frac{1}{2} t_{2}, i_{1}=\frac{1}{2} d_{1}+\frac{1}{2} t_{1}, k_{2}=\frac{1}{2} d_{2}, i_{2}=\frac{1}{2} d_{2}+\frac{1}{2} t_{2}, t_{3}=\frac{1}{2}, f_{1}=\frac{1}{2}+\frac{1}{2} t_{1}, k_{1}=\frac{1}{2} d_{1}$.

With the second option to describe midpoints the theses remain unchanged, and the elimination ideal of hypotheses and negation of theses is

$$
<\left(d_{1}^{2}+d_{2}^{2}\right)\left(-2 t_{1}+1+t_{1}^{2}+t_{2}^{2}\right)\left(t_{1}^{2}-2 t_{1} d_{1}+d_{2}^{2}+d_{1}^{2}-2 t_{2} d_{2}+t_{2}^{2}\right)>,
$$

meaning that the statement is generally true. That is, the projection, over the free variables, of the variety where the theses do not hold is reduced to (the geometric interpretation is done over the real plane)

- $d_{1}^{2}+d_{2}^{2}=0$, i.e. $d_{1}=d_{2}=0$, a degenerate case $D=A$, or

- $-2 t_{1}+1+t_{1}^{2}+t_{2}^{2}=0$, i.e. $t_{1}=1, t_{2}=0$, so $C=B$, or

- $t_{1}^{2}-2 t_{1} d_{1}+d_{2}^{2}+d_{1}^{2}-2 t_{2} d_{2}+t_{2}^{2}$, i.e. $d_{1}=t_{1}$ and $d_{2}=t_{2}$, a degenerate case $D=C$. 
Moreover, each of the theses belongs to the ideal of the hypotheses, extended with the negation of

$$
\left(d_{1}^{2}+d_{2}^{2}\right)\left(-2 t_{1}+1+t_{1}^{2}+t_{2}^{2}\right)\left(t_{1}^{2}-2 t_{1} d_{1}+d_{2}^{2}+d_{1}^{2}-2 t_{2} d_{2}+t_{2}^{2}\right)=0,
$$

as it can be checked by

$$
\begin{aligned}
& >(\mathrm{f} 1-\mathrm{t} 3) *(\mathrm{k} 2-\mathrm{i} 2)-(\mathrm{f} 2-\mathrm{t} 4) *(\mathrm{k} 1-\mathrm{i} 1) \text { in }<(0-\mathrm{t} 3)^{\wedge} 2+(0-\mathrm{t} 4)^{\wedge} 2-\left((1-\mathrm{t} 3)^{\wedge} 2+\right. \\
& \left.(0-t 4)^{\wedge} 2\right), t 4,(1-f 1)^{\wedge} 2+(0-f 2)^{\wedge} 2-\left((t 1-f 1)^{\wedge} 2+(t 2-f 2)^{\wedge} 2\right),(1-f 1) *(t 2- \\
& f 2)-(0-f 2) *(t 1-f 1),(t 1-i 1)^{\wedge} 2+(t 2-i 2)^{\wedge} 2-\left((d 1-i 1)^{\wedge} 2+(d 2-i 2)^{\wedge} 2\right) \text {, } \\
& (\mathrm{d} 1-\mathrm{i} 1) *(\mathrm{t} 2-\mathrm{i} 2)-(\mathrm{d} 2-\mathrm{i} 2) *(\mathrm{t} 1-\mathrm{i} 1),(\mathrm{d} 1-\mathrm{k} 1)^{\wedge} 2+(\mathrm{d} 2-\mathrm{k} 2)^{\wedge} 2-\left((0-\mathrm{k} 1)^{\wedge} 2+(0\right. \\
& \left.-\mathrm{k} 2)^{\wedge} 2\right),(\mathrm{d} 1-\mathrm{k} 1) *(0-\mathrm{k} 2)-(\mathrm{d} 2-\mathrm{k} 2) *(0-\mathrm{k} 1),\left(\mathrm{d} 1{ }^{\wedge} 2+\mathrm{d} 2^{\wedge} 2\right) *\left(-2 * \mathrm{t} 1+1+\mathrm{t} 1^{\wedge} 2+\right. \\
& \left.t 2^{\wedge} 2\right) *\left(t 1^{\wedge} 2-2 * t 1 * d 1+d 2^{\wedge} 2+d 1^{\wedge} 2-2 * t 2 * d 2+t 2^{\wedge} 2\right) * h-1>\text {; } \\
& \text { true }
\end{aligned}
$$

and

$$
\begin{aligned}
& >(k 1-t 3) *(f 2-i 2)-(k 2-t 4) *(f 1-i 1) \text { in }<(0-t 3)^{\wedge} 2+(0-t 4)^{\wedge} 2-\left((1-t 3)^{\wedge} 2+\right. \\
& \left.(0-t 4)^{\wedge} 2\right), t 4,(1-f 1)^{\wedge} 2+(0-f 2)^{\wedge} 2-\left((t 1-f 1)^{\wedge} 2+(t 2-f 2)^{\wedge} 2\right),(1-f 1) *(t 2- \\
& f 2)-(0-f 2) *(t 1-f 1),(t 1-i 1)^{\wedge} 2+(t 2-i 2)^{\wedge} 2-\left((d 1-i 1) \wedge 2+(d 2-i 2)^{\wedge} 2\right) \text {, } \\
& (\mathrm{d} 1-\mathrm{i} 1) *(\mathrm{t} 2-\mathrm{i} 2)-(\mathrm{d} 2-\mathrm{i} 2) *(\mathrm{t} 1-\mathrm{i} 1),(\mathrm{d} 1-\mathrm{k} 1)^{\wedge} 2+(\mathrm{d} 2-\mathrm{k} 2)^{\wedge} 2-\left((0-\mathrm{k} 1)^{\wedge} 2+(0\right. \\
& \left.-\mathrm{k} 2)^{\wedge} 2\right),(\mathrm{d} 1-\mathrm{k} 1) *(0-\mathrm{k} 2)-(\mathrm{d} 2-\mathrm{k} 2) *(0-\mathrm{k} 1),\left(\mathrm{d} 1 \wedge 2+\mathrm{d} 2^{\wedge} 2\right) *\left(-2 * \mathrm{t} 1+1+\mathrm{t} 1^{\wedge} 2+\right. \\
& \left.t 2^{\wedge} 2\right) *\left(t 1^{\wedge} 2-2 * t 1 * d 1+d 2^{\wedge} 2+d 1^{\wedge} 2-2 * t 2 * d 2+t 2 \wedge 2\right) * h-1>\text {; } \\
& \text { true }
\end{aligned}
$$

thus confirming that this extended statement is generally true. Even more, the computation

$$
\begin{aligned}
& >1 \text { in }<(0-t 3)^{\wedge} 2+(0-t 4)^{\wedge} 2-\left((1-t 3)^{\wedge} 2+(0-t 4)^{\wedge} 2\right), t 4,(1-f 1)^{\wedge} 2+(0-f 2)^{\wedge} 2 \\
& -\left((t 1-f 1)^{\wedge} 2+(t 2-f 2)^{\wedge} 2\right),(1-f 1) *(t 2-f 2)-(0-f 2) *(t 1-f 1),(t 1-i 1)^{\wedge} 2+ \\
& (t 2-i 2)^{\wedge} 2-\left((d 1-i 1)^{\wedge} 2+(d 2-i 2)^{\wedge} 2\right),(d 1-i 1) *(t 2-i 2)-(d 2-i 2) *(t 1-i 1) \text {, } \\
& (\mathrm{d} 1-\mathrm{k} 1)^{\wedge} 2+(\mathrm{d} 2-\mathrm{k} 2)^{\wedge} 2-\left((0-\mathrm{k} 1)^{\wedge} 2+(0-\mathrm{k} 2)^{\wedge} 2\right),(\mathrm{d} 1-\mathrm{k} 1) *(0-\mathrm{k} 2)-(\mathrm{d} 2-\mathrm{k} 2) \text { * } \\
& (0-k 1),(((f 1-t 3) *(k 2-i 2)-(f 2-t 4) *(k 1-i 1)) * t-1) *(((k 1-t 3) *(f 2-i 2) \\
& -(\mathrm{k} 2-\mathrm{t} 4) *(\mathrm{f} 1-\mathrm{i} 1)) * \mathrm{~s}-1),\left(\mathrm{d} 1^{\wedge} 2+\mathrm{d} 2^{\wedge} 2\right) *\left(-2 * t 1+1+\mathrm{t} 1^{\wedge} 2+\mathrm{t} 2^{\wedge} 2\right) *\left(\mathrm{t} 1^{\wedge} 2-2 *\right. \\
& \left.\mathrm{t} 1 * \mathrm{~d} 1+\mathrm{d} 2 \wedge 2+\mathrm{d} 1^{\wedge} 2-2 * t 2 * \mathrm{~d} 2+\mathrm{t} 2^{\wedge} 2\right) * \mathrm{~h}-1>\text {; } \\
& \text { true }
\end{aligned}
$$

shows that 1 is a combination of the ideal of extended hypotheses and the negation of the theses, remarking the absolute truth of this extended statement.

As an alternative, but less rigorous proof, since computing the above elimination ideal took several hours, it can be checked that the values of the depending coordinates do verify the theses:

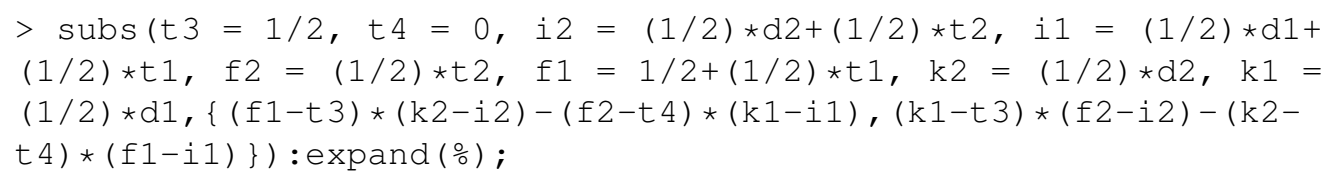

\subsection{The Converse Varignon theorem}

4.2.1. Converse Varignon: option a). Using option a) for the concept of midpoint, let us consider a parallelogram $A(0,0), B(1,0), C\left(t_{1}, t_{2}\right), D\left(d_{1}, d_{2}\right)$ such that $D C \| A B$ and $A D \| B C$ (Figure 3), that is, $d_{1} t_{2}=d_{2}\left(t_{1}-1\right), d_{2}=t_{2}$.

Fix a free point $E\left(t_{3}, t_{4}\right)$, build the line $E A$, and, on this line, the point $F\left(f_{1}, f_{2}\right)$ such that $A$ is the midpoint of segment $E F$, so $t_{3}=-f_{1}, t_{4}=-f_{2}$. Analogously, point $I\left(i_{1}, i_{2}\right)$ such that $B$ is $F I$ midpoint $\left(f_{1}+i_{1}=2, f_{2}=-i_{2}\right)$, and point $K\left(k_{1}, k_{2}\right)$ such that $C$ is $I K$ midpoint $\left(k_{1}+i_{1}=2 t_{1}, k_{2}+i_{2}=2 t_{2}\right)$.

Thus, the 8 hypotheses are

$$
d_{1} t_{2}-d_{2}\left(t_{1}-1\right), d_{2}-t_{2}, t_{3}+f_{1}, t_{4}+f_{2}, f_{1}+i_{1}-2, f_{2}+i_{2}, k_{1}+i_{1}-2 t_{1}, k_{2}+i_{2}-2 t_{2} \text {. }
$$




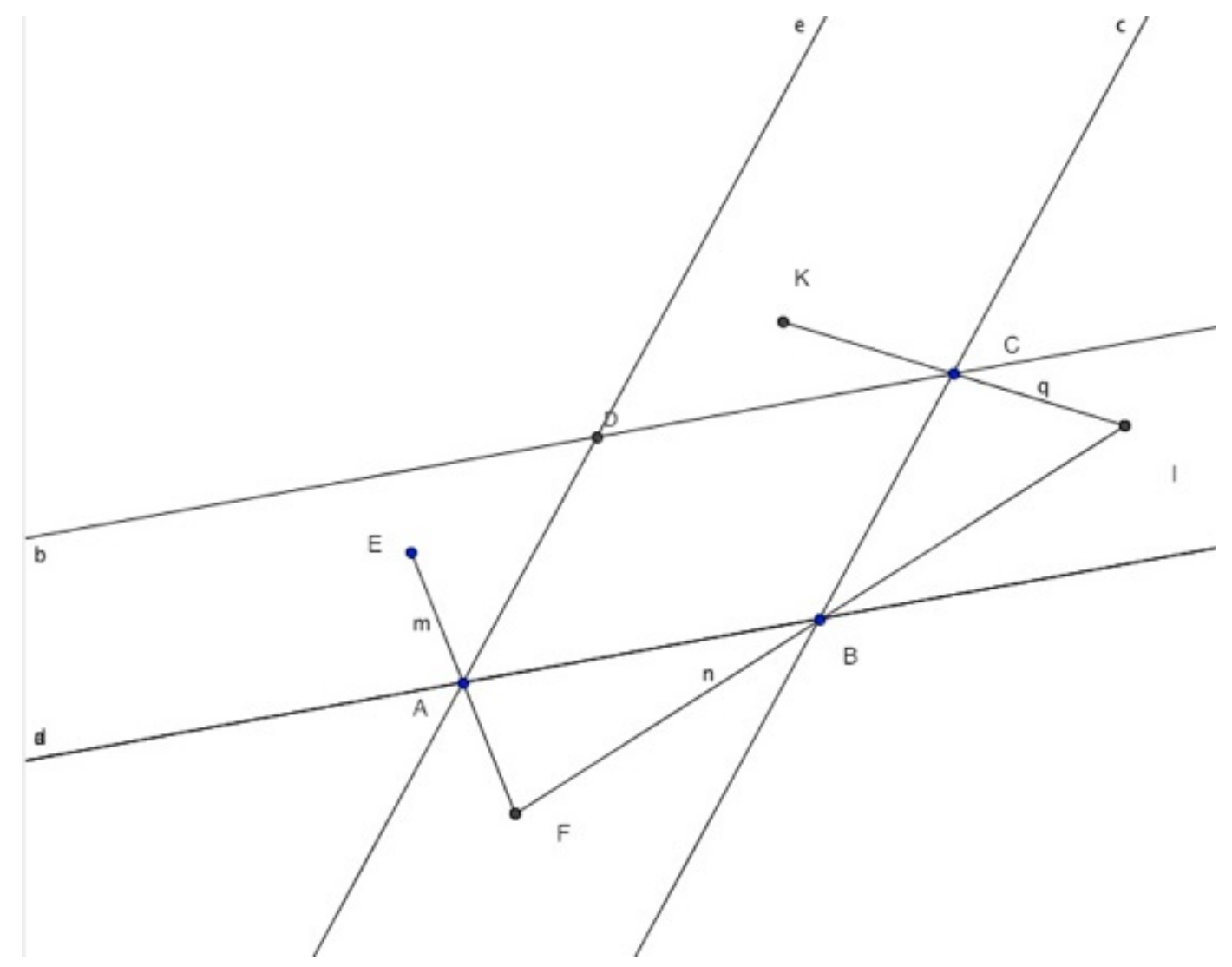

FIgURE 3. Using option a) for Converse Varignon theorem

The construction involves the 12 variables $\left\{t_{1}, t_{2}, t_{3}, t_{4}, d_{1}, d_{2}, f_{1}, f_{2}, i_{1}, i_{2}, k_{1}, k_{2}\right\}$; the dimension of the construction is 4

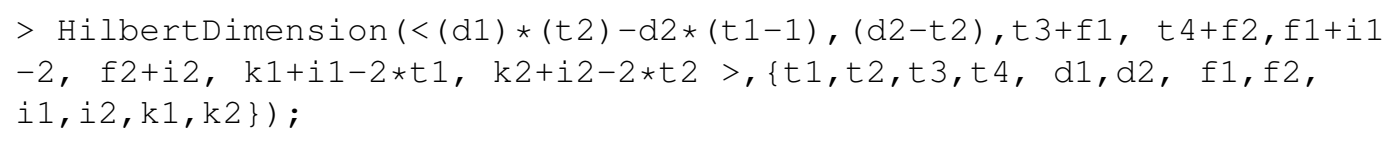

The first four variables are free

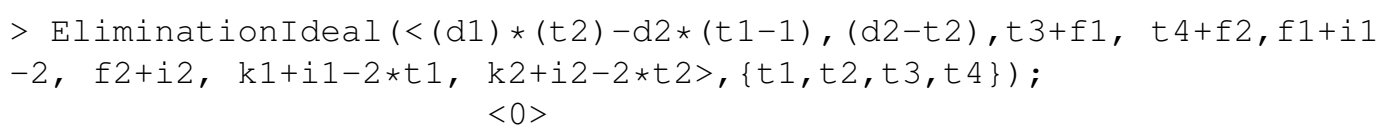

while the remaining ones are uniquely determined if values are assigned to the free variables $\left\{t_{1}, t_{2}, t_{3}, t_{4}\right\}$

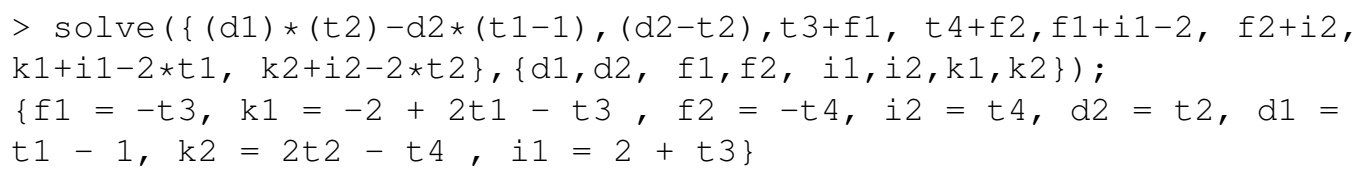

We claim that $D$ is the midpoint of segment $E K$, i.e. the simultaneous vanishing of $k_{1}+t_{3}-$ $2 d_{1}$ and $k_{2}+t_{4}-2 d_{2}$. We add these two theses to the hypotheses and see what are the consequences of this claim: 


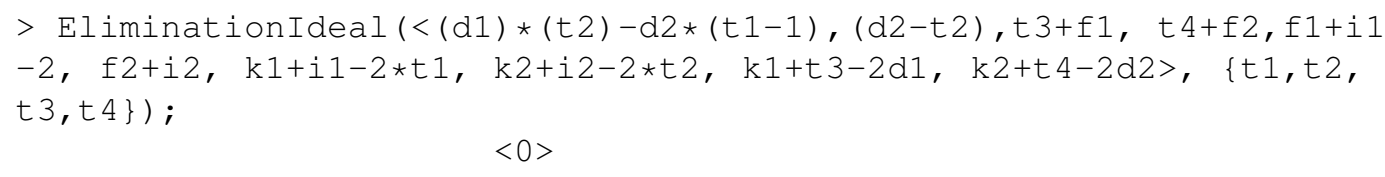

So, the consequence (on the space of free points) of our claim is the whole space, i.e. all values of $t_{1}, t_{2}, t_{3}, t_{4}$ are on the closure of the projection of the set of points verifying the hypotheses and the theses. In other words, for almost every value of $t_{1}, t_{2}, t_{3}, t_{4}$ there is a value of $d_{1}, d_{2}, f_{1}, f_{2}, i_{1}, i_{2}, k_{1}, k_{2}$ verifying that the midpoints of the quadrilateral $E F I K$ form the parallelogram $A B C D$.

In principle, from the last elimination result, one can just conclude that the statement is not generally false, because for almost each value of the free parameters there is a value of the depending variables $d_{1}, d_{2}, i_{1}, i_{2}, k_{1}, k_{2}$, so that the statement is true. But it could happen, in principle, that there is also a different value of $d_{1}, d_{2}, f_{1}, f_{2}, i_{1}, i_{2}, k_{1}, k_{2}$ where the statement is false; we know in this particular case that this can not happen, since there is only one value of the depending variables for each value of the free ones; but this kind of argument depends on the Solve command, which is not easy to handle or canonical, in general, and thus it is not used as a standard in the dynamic geometry proving routines.

So, let us see by a different, more general method, that our statement is generally true by considering the collection of hypotheses and the negation of the theses:

$$
\left(\left(k_{1}+t_{3}-2 d_{1}\right) t-1\right)\left(\left(k_{2}+t_{4}-2 d_{2}\right) s-1\right)=0 .
$$

Then, we project, over the free parameter space, the variety given by the hypotheses and the negation of the theses

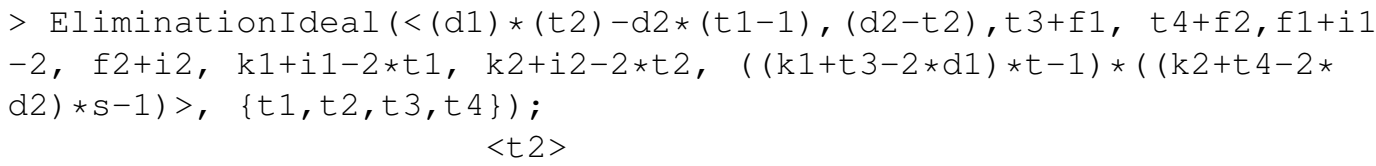

The result means that if $t_{2} \neq 0$, then the statement is true, i.e. there is no solution to the set of hypotheses and negation of theses

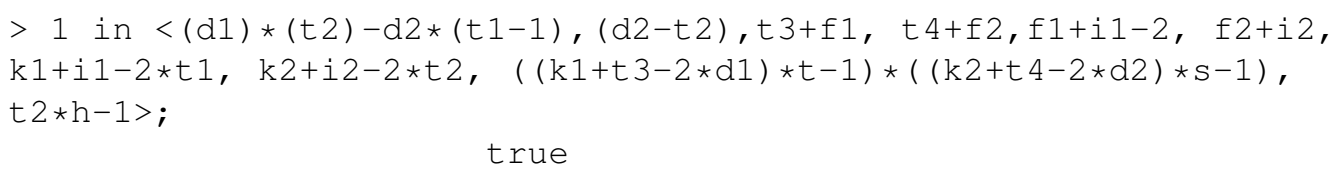

\section{true}

In conclusion, with this formulation the statement is not generally false and it is generally true.

4.2.2. Converse Varignon: option b). Proving it is not generally false. Using midpoint concept b), the conditions on the parallelogram $A(0,0), B(1,0), C\left(t_{1}, t_{2}\right), D\left(d_{1}, d_{2}\right)$ such that $D C \| A B$ and $A D \| B C$ remain as above $d_{1} t_{2}=d_{2}\left(t_{1}-1\right), d_{2}=t_{2}$. Next we fix a free point $E\left(t_{3}, t_{4}\right)$, build the line $E A$, and, on this line, the point $F\left(f_{1}, f_{2}\right)$ such that $A$ is the midpoint of segment $E F$. Here $A$ is now the center of a circle passing through $E$ and $F$, and $F$ is in the line $E A$ : $f_{1}^{2}+f_{2}^{2}=t_{3}^{2}+t_{4}^{2}, t_{3} f_{2}=t_{4} f_{1}$. Idem, point $I\left(i_{1}, i_{2}\right)$ such that $B$ is the midpoint of segment $F I$ : $\left(i_{1}-1\right)^{2}+i_{2}^{2}=\left(f_{1}-1\right)^{2}+f_{2}^{2},\left(f_{1}-1\right) i_{2}=f_{2}\left(i_{1}-1\right)$. Idem, point $K\left(k_{1}, k_{2}\right)$ such that $C$ is the midpoint of $\left.I K:\left(k_{1}-t_{1}\right)^{2}+\left(k_{2}-t_{2}\right)^{2}=\left(i_{1}-t 1\right)^{2}+i_{2}-t_{2}\right)^{2},\left(k_{1}-t_{1}\right)\left(i_{2}-t_{2}\right)=\left(k_{2}-t_{2}\right)\left(\left(i_{1}-t_{1}\right)\right.$.

The construction involves 12 variables $\left\{t_{1}, t_{2}, t_{3}, t_{4}, d_{1}, d_{2}, f_{1}, f_{2}, i_{1}, i_{2}, k_{1}, k_{2}\right\}$. It is of dimension 4

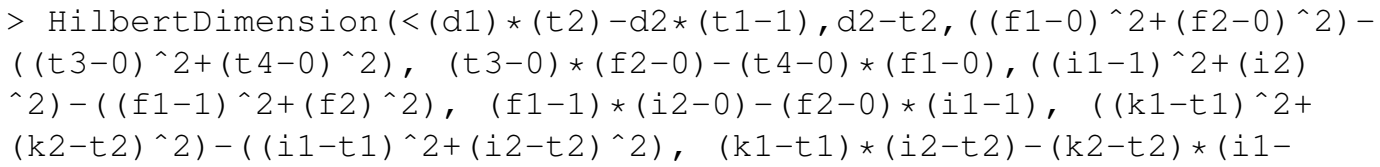




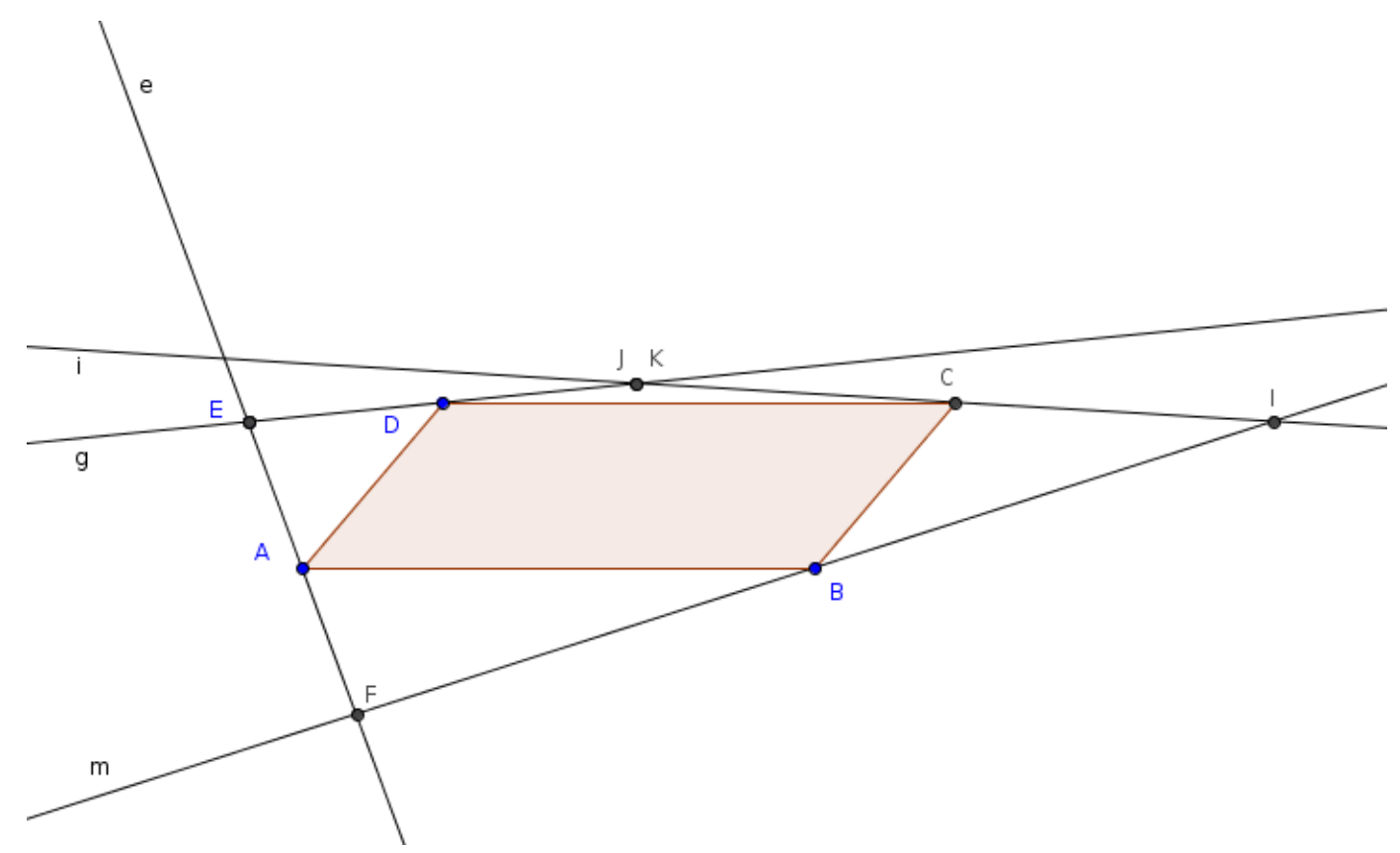

FIGURE 4. Using option b) with Converse Varignon theorem

$t 1)>,\{t 1, t 2, t 3, t 4, d 1, d 2, f 1, f 2, i 1, i 2, k 1, k 2\})$;

where the first four variables are free

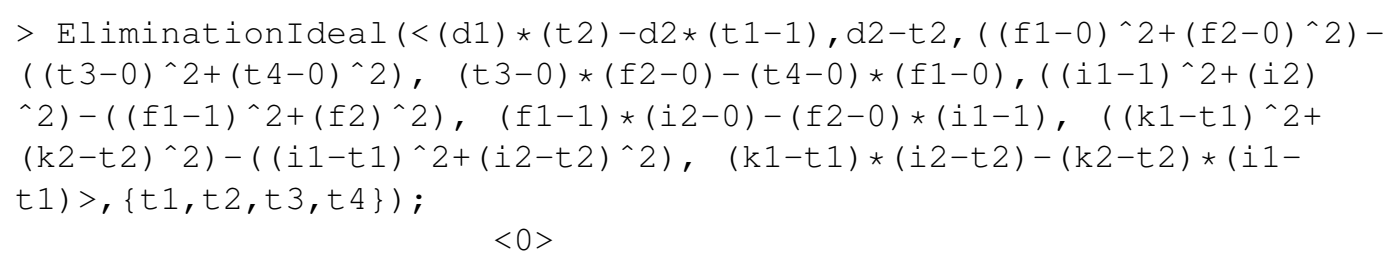

and the remaining variables are finitely determined. There are eight possible options for every $\left\{t_{1}, t_{2}, t_{3}, t_{4}\right\}$ : two for point $E$; for each of them, two for point $I$, and, for every value of $I$, two for point $K$

$$
\begin{aligned}
& >\mathrm{S}:=\mathrm{solve}\left(\left\{(\mathrm{d} 1) *(\mathrm{t} 2)-\mathrm{d} 2 *(\mathrm{t} 1-1), \mathrm{d} 2-\mathrm{t} 2,\left((\mathrm{f} 1-0)^{\wedge} 2+(\mathrm{f} 2-0)^{\wedge} 2\right)-((\mathrm{t} 3-0)\right.\right. \\
& \left.\wedge 2+(t 4-0)^{\wedge} 2\right),(t 3-0) *(f 2-0)-(t 4-0) *(f 1-0),\left((i 1-1)^{\wedge} 2+(i 2)^{\wedge} 2\right)-((f 1 \\
& \left.-1)^{\wedge} 2+(\mathrm{f} 2)^{\wedge} 2\right),(\mathrm{f} 1-1) *(i 2-0)-(\mathrm{f} 2-0) *(\mathrm{i} 1-1),\left((\mathrm{k} 1-\mathrm{t} 1)^{\wedge} 2+(\mathrm{k} 2-\mathrm{t} 2)\right. \\
& \text { ^2) }-((i 1-t 1) \wedge 2+(i 2-t 2) \wedge 2),(k 1-t 1) *(i 2-t 2)-(k 2-t 2) *(i 1-t 1)\},\{d 1, \\
& \mathrm{d} 2, \mathrm{f1}, \mathrm{f2}, \mathrm{i} 1, \mathrm{i} 2, \mathrm{k} 1, \mathrm{k} 2\}) ; \operatorname{nops}([\mathrm{S}]) \text {; } \\
& \{\mathrm{d} 1=\mathrm{t} 1-1, \mathrm{~d} 2=\mathrm{t} 2, \mathrm{fl}=\mathrm{t} 3, \mathrm{f2}=\mathrm{t} 4, \mathrm{i} 1=\mathrm{t} 3, \mathrm{i} 2=\mathrm{t} 4, \mathrm{k} 1=\mathrm{t} 3, \\
& \mathrm{k} 2=\mathrm{t} 4\},\{\mathrm{d} 1=\mathrm{t} 1-1, \mathrm{~d} 2=\mathrm{t} 2, \mathrm{f} 1=\mathrm{t} 3, \mathrm{f2}=\mathrm{t} 4, \mathrm{i} 1=\mathrm{t} 3, \mathrm{i} 2=\mathrm{t} 4, \\
& \mathrm{k} 1=2 * t 1-\mathrm{t} 3, \mathrm{k} 2=2 * t 2-\mathrm{t} 4\}, \quad\{\mathrm{d} 1=\mathrm{t} 1-1, \mathrm{~d} 2=\mathrm{t} 2, \mathrm{f} 1=\mathrm{t} 3, \mathrm{f} 2 \\
& =\mathrm{t} 4, \mathrm{i} 1=-\mathrm{t} 3+2, \mathrm{i} 2=-\mathrm{t} 4, \mathrm{k} 1=-\mathrm{t} 3+2, \mathrm{k} 2=-\mathrm{t} 4\},\{\mathrm{d} 1=\mathrm{t} 1-1, \mathrm{~d} 2 \\
& =t 2, \mathrm{fl}=\mathrm{t} 3, \mathrm{f} 2=\mathrm{t} 4, \mathrm{i} 1=-\mathrm{t} 3+2, \mathrm{i} 2=-\mathrm{t} 4, \mathrm{k} 1=2 * t 1+\mathrm{t} 3-2, \mathrm{k} 2= \\
& 2 * t 2+t 4\},\{d 1=t 1-1, d 2=t 2, f 1=-t 3, f 2=-t 4, i 1=-t 3, \\
& \mathrm{i} 2=-\mathrm{t} 4, \mathrm{k} 1=-\mathrm{t} 3, \mathrm{k} 2=-\mathrm{t} 4\}, \quad \mathrm{d} 1=\mathrm{t} 1-1, \mathrm{~d} 2=\mathrm{t} 2, \mathrm{f} 1=-\mathrm{t} 3, \\
& \mathrm{f} 2=-\mathrm{t} 4, \mathrm{i} 1=-\mathrm{t} 3, \mathrm{i} 2=-\mathrm{t} 4, \mathrm{k} 1=2 * t 1+\mathrm{t} 3, \mathrm{k} 2=2 * \mathrm{t} 2+\mathrm{t} 4\} \text {, }
\end{aligned}
$$




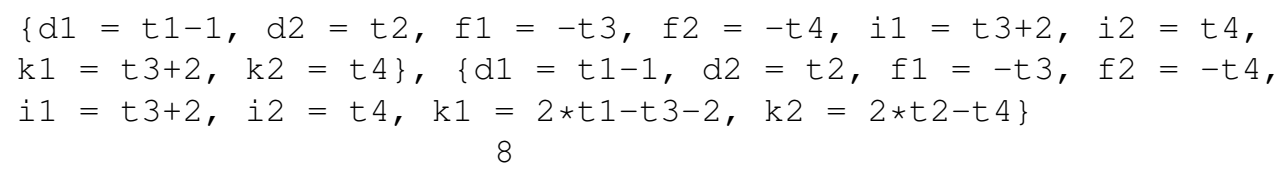

Recall that the thesis is that $D$ is the midpoint of segment $K E$, that is, $k_{1}+t_{3}=2 d_{1}$ and $k_{2}+$ $t_{4}=2 d_{2}$. We will add these polynomials to the hypotheses and will see what are the consequence of this claim. Note that there is a hypothesis (on parallelism) stating that $d_{2}=t_{2}$. Thus the ideal of hypotheses and theses is isomorphic to the ideal obtained replacing $d_{2}$ by $t_{2}$. In this way we define an equivalent, simpler, ideal with less variables and easier to handle:

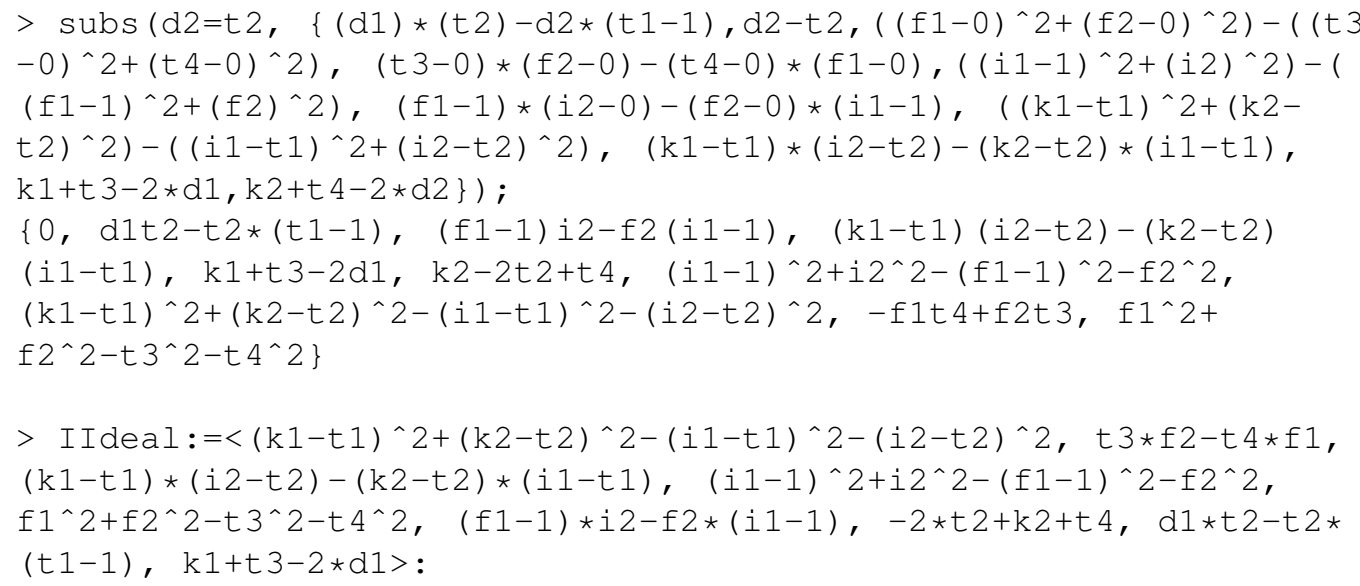

In order to simplify the costly elimination process, we realize that I Ideal contains a polynomial $d_{1} t_{2}-t_{2}\left(t_{1}-1\right)$ that factors as $t_{2}$ times $d_{1}-t_{1}+1$. So, we replace in I Ideal the polynomial $d_{1} t_{2}-t_{2}\left(t_{1}-1\right)$ by $d_{1}-t_{1}+1$, defining IdealA; likewise, replacing in I Ideal $d_{1} t_{2}-t_{2}\left(t_{1}-1\right)$ by $t_{2}$ we define IdealB. It can be checked that the intersection of IdealA and IdealB is equal to IIdeal

$>$ IdealC:=Intersect (IdealA, IdealB) : IdealContainment (IdealC, IIdeal, IdealC);

\section{true}

So, the elimination of IIdeal over the variables $\left\{t_{1}, t_{2}, t_{3}, t_{4}\right\}$ is $\langle 0\rangle$ because the elimination of IdealA is $\langle 0\rangle$ and the elimination of IdealB is $\left\langle t_{2}\right\rangle$.

$>$ EliminationIdeal (IdealA, $\{t 1, t 2, t 3, t 4\}$ ); EliminationIdeal (IdealB, $\{t 1, t 2, t 3, t 4\}) ;$

$<0>$
$<t 2>$
$>$ EliminationIdeal(IIdeal, $\{t 1, t 2, t 3, t 4\}) ;$
$<0>$

This means

- the statement is not generally false, because for almost each value of the free parameters there is a value of the depending variables $d_{1}, f_{1}, f_{2}, i_{1}, i_{2}, k_{1}, k_{2}$ where the theses and the hypotheses hold, so that the statement is true; but it could happen that there is also a different value of $d_{1}, f_{1}, f_{2}, i_{1}, i_{2}, k_{1}, k_{2}$ where the statement is false. We know in this particular case, by solving the system given by IIdeal that this can not happen, since there is only one value of the depending variables for each value of the free ones. But this kind of argument depends on the Solve command, which we will like to avoid for the reasons expressed above. 


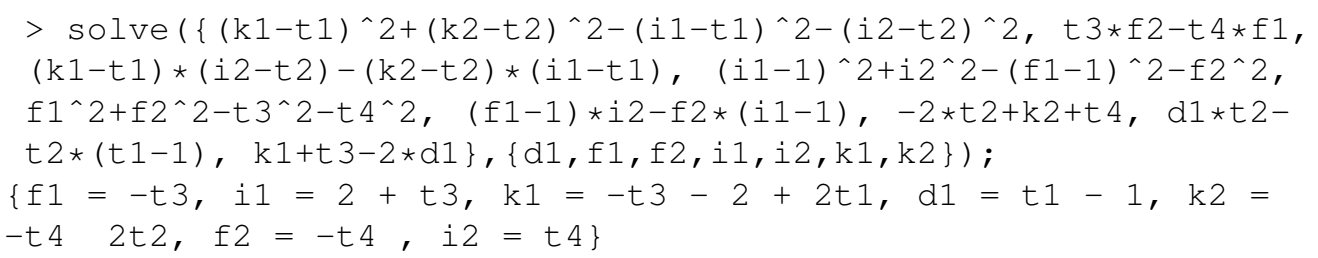

- this one value is, precisely, the one that corresponds to the intuitive idea about $F, I, K \ldots$

- remark that the elimination of IIdeal coincides with that of IdealA; again, the above Solve output for IIdeal coincides with that for IdealA, but there is not general solution for IdealB (a degenerate case, with $t_{2}=0$, the parallelogram degenerates to a line), getting an empty output to the following command:

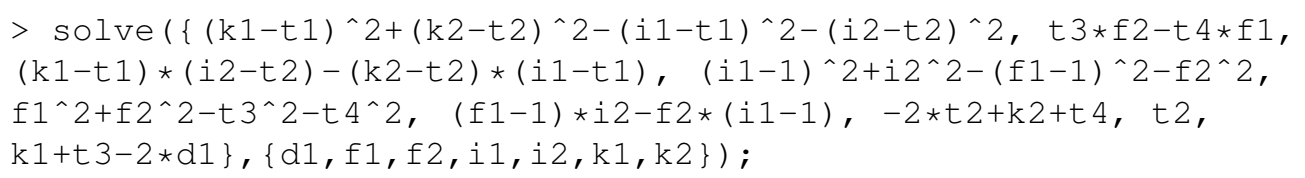

4.2.3. Converse Varignon: option b). Proving it is not generally true. Unfortunately, we can not prove that this formulation of the Converse Varignon statement is generally true. In fact, let us try to see that it is not generally true, by considering the collection of hypotheses and the negation of theses $\left(\left(\left(k_{1}+t_{3}-2 d_{1}\right) t-1\right)\left(\left(k_{2}+t_{4}-2 d_{2}\right) s-1\right)=0\right)$. Then, we project over the free parameter space the variety given by the hypotheses and the negation of the theses, by eliminating all variables except $t_{1}, t_{2}, t_{3}, t_{4}$. If this elimination is 0 , then it is not generally true, because it means that for almost all values of the free points $C, E$, there are values of the remaining variables so that the thesis is not true. Taking in consideration that we have already proved the statement is not generally false, proving it is not generally true, it would mean that for almost all positions of the free points $C, E$, there are values of the remaining points such that the thesis holds (because the statement is not generally false), but there are also values of such points where the thesis does not hold (because it is not generally true). Let us see if we can achieve proving it is not generally true, by

$$
\text { Elimination (Ideal of Hypotheses +Negation of Theses, }\left\{t_{1}, t_{2}, t_{3}, t_{4}\right\} \text { ). }
$$

But this elimination, directly, is too involved concerning time and memory. Let us attempt to simplify it, as above, by, first, substituting $d_{2}=t_{2}$ in all polynomials of the ideal of hypotheses and negation of theses, yielding IdealN. Then we split in two factors the generator $d_{1} t_{2}-t_{2}\left(t_{1}-1\right)$ obtaining factor $d_{1}-\left(t_{1}-1\right)$ and factor $t_{2}$. Likewise, we split in two factors the generator $\left(\left(k_{1}+t_{3}-\right.\right.$ $\left.\left.2 d_{1}\right) t-1\right)\left(\left(k_{2}+t_{4}-2 d_{2}\right) s-1\right)$. Thus, combining the four resulting factors, we build four ideals. IdealP 1 and IdealP 2 , both with factor $d_{1}-\left(t_{1}-1\right)$ and with factors $\left(\left(k_{1}+t_{3}-2 d_{1}\right) t-1\right)$ or $\left.\left(k_{2}+t_{4}-2 d_{2}\right) s-1\right)$, respectively. Same, IdealQ1 and IdealQ2, both with factor $t_{2}$ and with factors $\left(\left(k_{1}+t_{3}-2 d_{1}\right) t-1\right)$ or $\left.\left(k_{2}+t_{4}-2 d_{2}\right) s-1\right)$. IdealR is the intersection of the four ideals. Obviously, IdealN is contained in each of the four ideals, and, thus, IdealN is contained in IdealR, and the converse is also true (by brute force computation with Maple, via IdealContainment command). Thus, IdealR is equal to IdealN, and the elimination in IdealN can be carried out by eliminating in IdealP1,IdealP2,IdealQ1,IdealQ2, and then finding the intersection of elimination.

Note that the elimination in IdealQ1 and IdealQ2

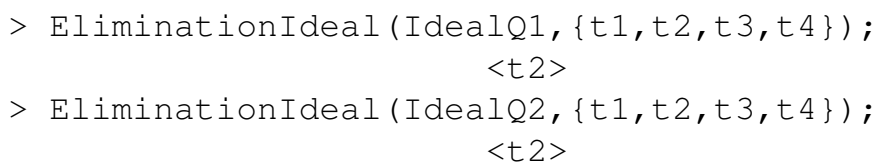


shows that the elimination applied to $I$ dea $I \mathrm{~N}$ is also contained in $\left\langle t_{2}\right\rangle$. We are not able to eliminate IdealP1, IdealP2 with Maple but it is so with Sage:

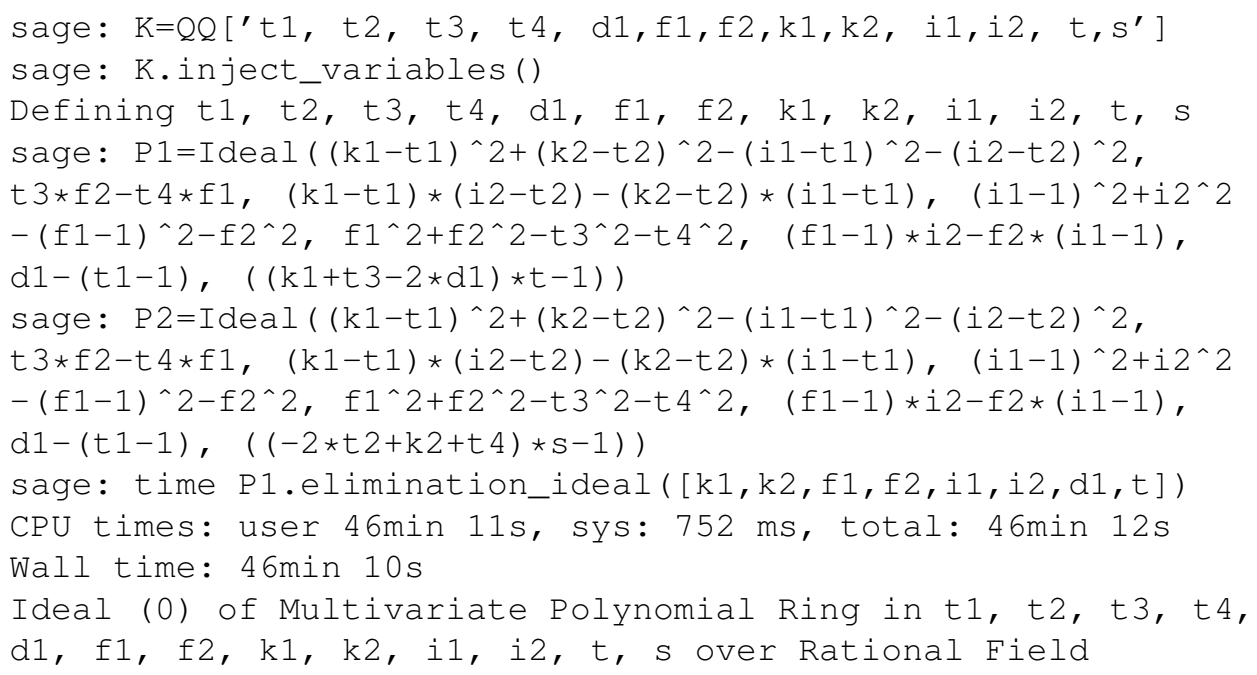

Thus, the statement would be not generally true.

4.2.4. Converse Varignon: option b). Learning from failure. Yet, there is an indirect way of proving -within Maple- that the statement is not generally true. Namely, we ask Maple to solve the system of equations given by the hypotheses and the negation of theses, so that the constrained variables $d_{1}, f_{1}, f_{2}, i_{1}, i_{2}, k_{1}, k_{2}, t, s$ (recall we have applied the equality $d_{2}=t_{2}$ and, thus, there are no terms in the variable $d_{2}$ in the system of equations) are solved in terms of the free variables $t_{1}, t_{2}, t_{3}, t_{4}$. If for almost all (i.e. all except for a closed set in the space of $t_{1}, t_{2}, t_{3}, t_{4}$ ) values of the free variables there is a solution to the system of equations given by the hypotheses and the negation of theses, it is clear that the projection of its solution set over the $t_{1}, t_{2}, t_{3}, t_{4}$-space will be almost all that space (i.e. the elimination ideal will be $\langle 0\rangle$, since 0 is the only equation verified by the closure of the projection, that is, by the whole $t_{1}, t_{2}, t_{3}, t_{4}$-space).

We can see that, in fact, for every value of $t_{1}, t_{2}, t_{3}, t_{4}$ (except for some values that would vanish some denominators), there are 13 different values of $d_{1}, d_{2}, i_{1}, i_{2}, k_{1}, k_{2}, t, s$ in the system of equations given by the hypotheses and the negation of theses:

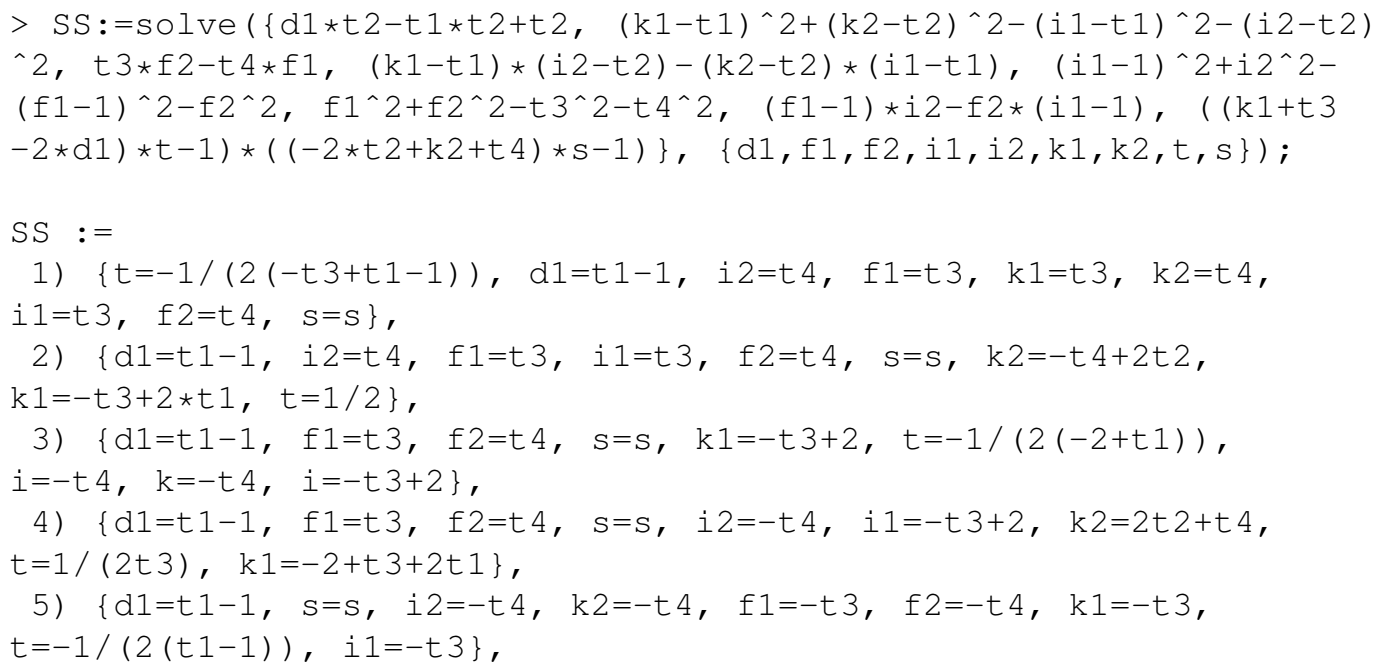


6) $\{\mathrm{d} 1=\mathrm{t} 1-1, \mathrm{~s}=\mathrm{s}, \mathrm{i} 2=-\mathrm{t} 4, \mathrm{k} 2=2 \mathrm{t} 2+\mathrm{t} 4, \mathrm{f} 1=-\mathrm{t} 3, \mathrm{f} 2=-\mathrm{t} 4, \mathrm{i} 1=-\mathrm{t} 3$,

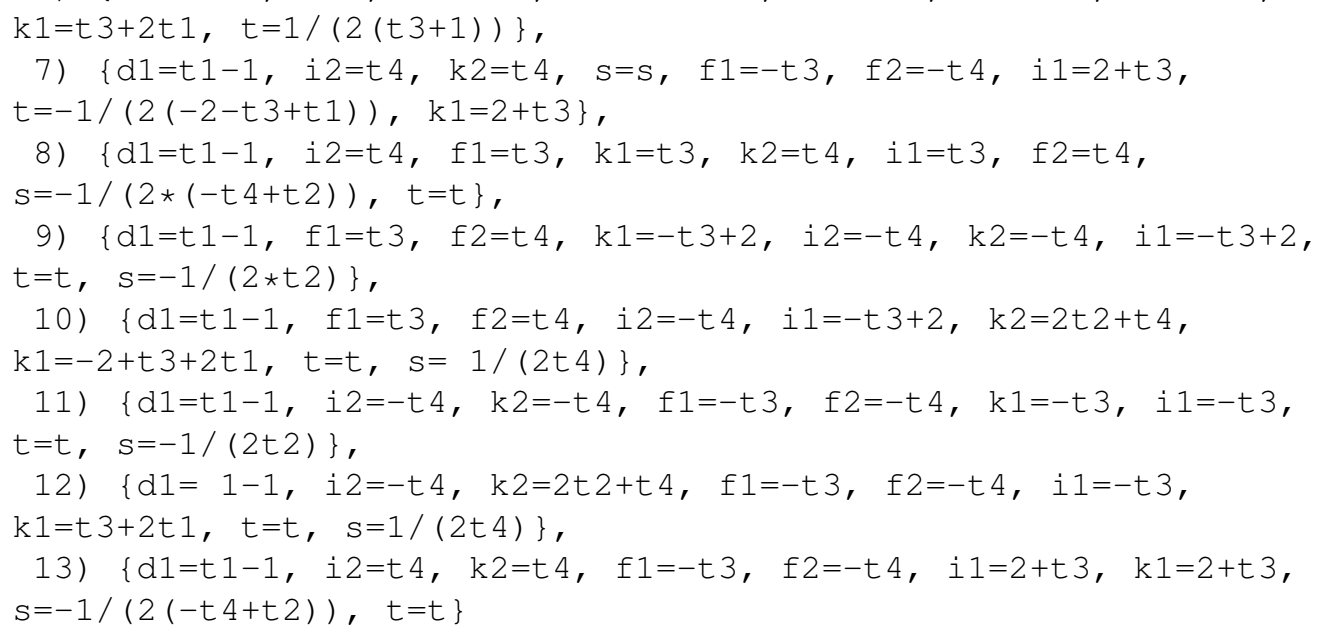

Why 13 solutions? We have seen that for every $t_{1}, t_{2}, t_{3}, t_{4}$ there are 8 different values of the remaining coordinates (i.e. of the cartesian product of the coordinates of the poins $F \times K \times I$ ). Let us repeat that output $S$ here below, identifying each of the blocks with a number from 1 to 8 :

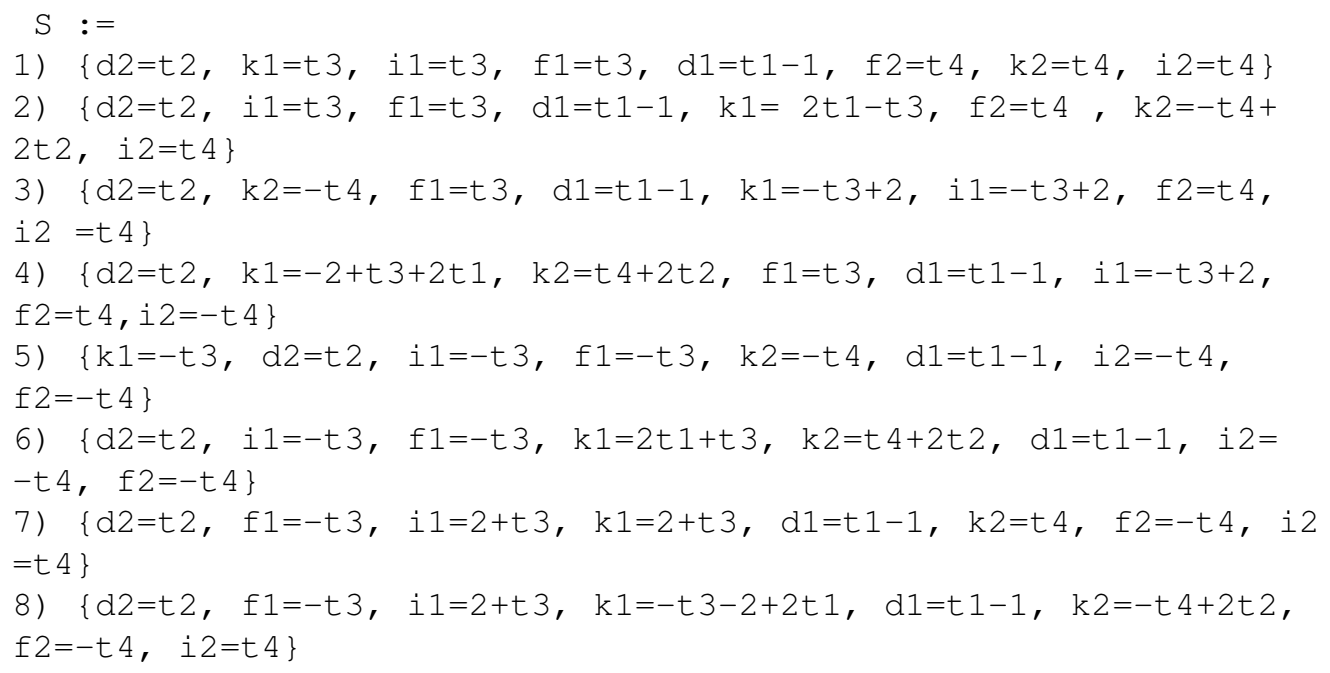

Recall that number 8) corresponds to the values of $F, K, I$ verifying the theses. So, the values of $F, K, I$ in $S S$ must come from one of the other blocks of $S$, say, 1) to 7).

In fact we can easily verify that the 13 blocks of $S S$ can be described as follows:

- twelve blocks of $S S$ correspond to the blocks 1), 3), 4), 5), 6), 7) of $S$ regarding the values of $d_{1}, f_{1}, f_{2}, i_{1}, i_{2}, k_{1}, k_{2}$. In all cases $d_{2}=t_{2}$ and $d_{1}=t_{1}-1$. Given these values of $d_{1}, f_{1}, f_{2}, i_{1}, i_{2}, k_{1}, k_{2}$, then the value of $t$ is, automatically, $t=1 /\left(\left(k_{1}+t_{3}-2 d 1\right)-\right.$ because the denominator is not identically zero- and $s$ can take any value; this description includes the first six blocks of $S S$ above. Then, there are other six blocks for the same values of $d_{1}, f_{1}, f_{2}, i_{1}, i_{2}, k_{1}, k_{2}$, where $t$ takes any value and $s$ is automatically the value of $s=1 /\left(k_{2}+t_{4}-2 d_{2}\right)$.

- a thirteenth block where $d_{1}, f_{1}, f_{2}, i_{1}, i_{2}, k_{1}, k_{2}$ correspond to block number 2) of $S$, but where $-2 t_{2}+k_{2}+t_{4}$ is then identically zero and $k_{1}+t_{3}-2 d_{1}$ is equal to $2 t_{1}-2 d_{1}$, so equal to 2 


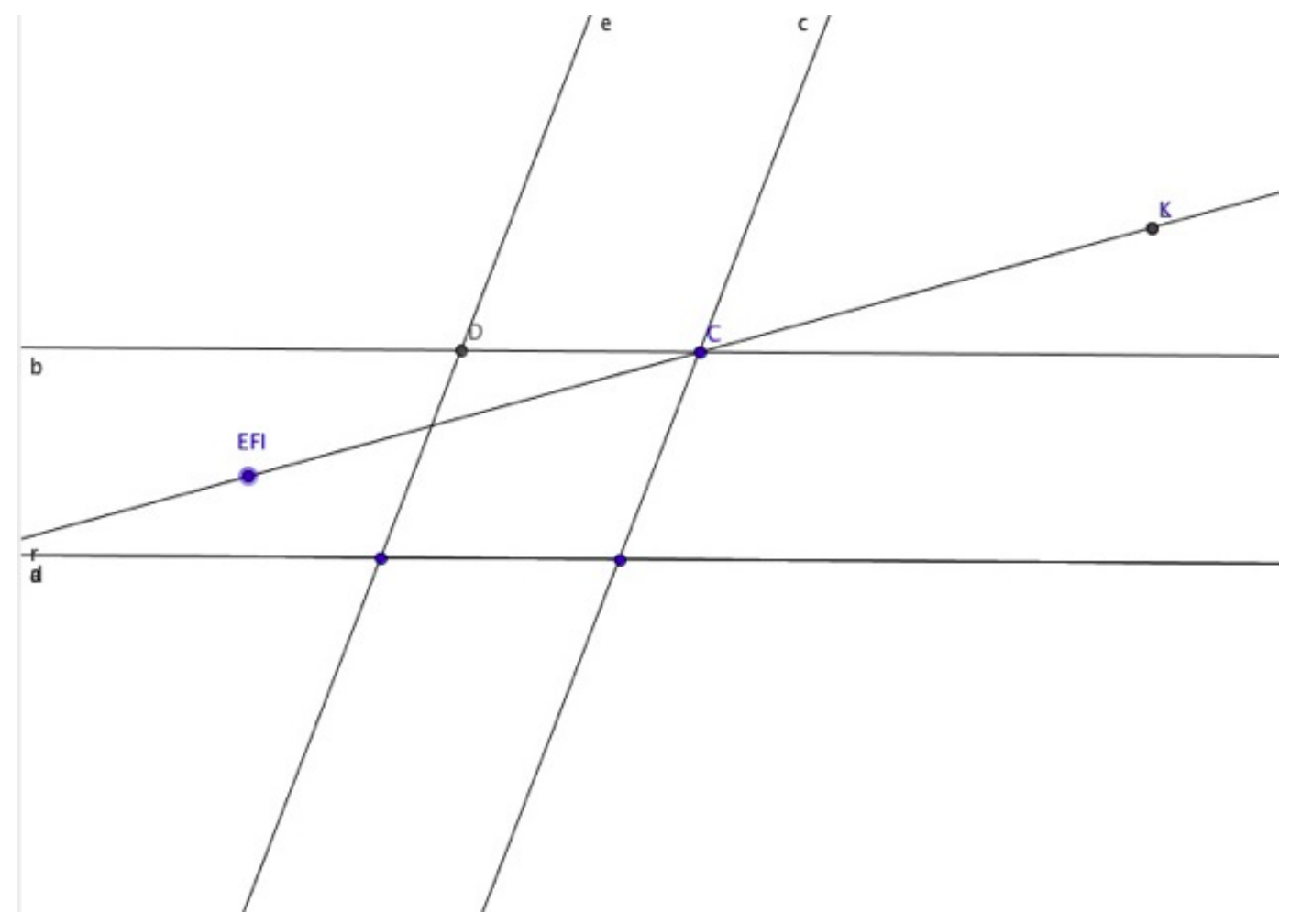

FIGURE 5. Learning from failure

( $t=1 / 2$ ), because $d_{1}=t_{1}-1 ;-$ so one the two theses is true- but where the other thesis does not hold, so $s$ is any value.

It is remarkable (probably it could be formulated as a new geometric statement) this asymmetry, the fact that there is only one instance of the construction verifying precisely one of the thesis (the one about the $y$ coordinate) and violating the other (and there is not one instance verifying the thesis about the $x$ coordinate and violating the other).

In fact, the meaning of the block of values for the depending variables

$\mathrm{k} 2=-\mathrm{t} 4+2 * t 2, i 2=t 4, \mathrm{f} 1=\mathrm{t} 3, \mathrm{k} 1=-\mathrm{t} 3+2 * t 1, \mathrm{il}=\mathrm{t} 3, \mathrm{f} 2=\mathrm{t} 4$

is, essentially that $E=F=I$, so that $K$ is actually the symmetrical of $E$ respect to $C$ (see Fig. $5)$, so that $t_{1}$ is $\left(k_{1}+t_{3}\right) / 2$ and $t_{2}$ is $\left(k_{2}+t_{4}\right) / 2$. So, now the theses are that $D$ is the midpoint of $K$ and $E$, so that $d_{2}=\left(k_{2}+t_{4}\right) / 2$, and $d_{1}=\left(k_{1}+t_{3}\right) / 2$. Obviously, the first equality, bearing in mind that $d_{2}=t_{2}$ in all the hypotheses, it holds because is part of the block description; the second equality does not, because, bearing in mind the block hypotheses, $d_{1}=\left(k_{1}+t_{3}\right) / 2$ is equivalent to $d_{1}=t_{1}, \ldots$, and this is not true, since $d_{1}=t_{1}-1$ in the construction, if $t_{2}$ is not zero. In other words, the thesis here is that $C=D$, and it is true that the $y$ coordinate of $C$ is equal to the $y$ coordinate of $D$, but not the $x$ coordinate.

In conclusion, the statement is not generally false, because for one interpretation of the construction both theses hold, but it is not generally true because for seven other interpretations of the construction none of the theses hold (in six cases times two, i.e., 12) or just one thesis does not hold (in the only one remaining case). This could be considered as the discovery of a (subtle) new geometric fact.... 


\title{
5. Conclusion
}

A detailed study of the theorem of Varignon has been performed. In a graphic environment, as the one provided by GeoGebra, the Varignon parallelogram requires for its specification a thorough understanding of the midpoint definition inside the system. We show that depending on the used midpoint definition, the statement can be declared generally true if the native GeoGebra midpoint command is used. Nevertheless, if the midpoint is given and the user must construct one of the endpoints (as in the converse Varignon statement), the computation can become very involved (for lack of a standard GeoGebra protocol) and the Varignon conclusion is generally true for the direct case, while it is neither generally true nor generally false for the converse case. Guessing complementary conditions to avoid falling in this confusing circumstance (without performing a primary decomposition, something not realistic in terms of required computing time and memory) is, sometimes intuitive, sometimes very complicated, yielding, if achieved, to the discovery of new geometric facts (such as the ones expressed by the different blocks in the above discussion). We think that these reflections should be carefully considered when designing user interfaces for massive use of theorem proving features in popular dynamic geometry programs, such as GeoGebra.

\section{References}

[1] Botana, F., Kovács, Z.: A Singular web service for geometric computations. Ann. Math. Artif. Intel. 74, 359-370 (2015)

[2] Botana, F., Hohenwarter, M., Janičić, J., Kovács, Z., Petrović, I., Recio, T., Weitzhofer, S.: Automated theorem proving in GeoGebra: Current achievements. J. Autom. Reason 55, 39-59 (2015)

[3] Chou, S.C.: Mechanical Geometry Theorem Proving. Reidel, Dordrecht (1988)

[4] Dalzotto, G., Recio, T.: On protocols for the automated discovery of theorems in elementary geometry. J. Autom. Reason 43, 203-236 (2009)

[5] Recio, T., Vélez, M.P.: Automatic discovery of theorems in elementary geometry. J. Autom. Reason 23, 63-82 (1999)

[6] Recio, T., Sterk, H., Vélez, M.P.: Project: Automatic geometry theorem proving. In: Cohen, A., Cuipers, H., Sterk, H. (eds.) Some Tapas of Computer Algebra pp. 276-296. Algorithms and Computations in Mathematics, Vol. 4, Springer, Heidelberg (1998)

\section{Acknowledgment}

Authors partially supported by the Spanish "Ministerio de Economía y Competitividad" and by the European Regional Development Fund (ERDF), under the Project MTM2014-54141-P.

\author{
Francisco Botana \\ Depto. de Matemática Aplicada I \\ University of Vigo \\ EE Forestal \\ 36005 Pontevedra \\ Spain \\ e-mail: fbotana@uvigo.es \\ Tomas Recio \\ Depto. de Matemáticas, Estadística y Computación \\ University of Cantabria \\ Avenida Los Castros \\ 39071 Santander \\ Spain \\ e-mail: tomas.recio@unican.es
}

